UCRL-JC-112349

PREPRINT

\title{
Opacity Measurements in Shock-Generated Argon Plasmas
}

\author{
David Erskine
}

This paper was prepared for submittal to the Proceedings of the Joint AIRAPT/APS Conference

Colorado Springs, $\mathrm{CO}$

June 28-July 2, 1993

July 1993

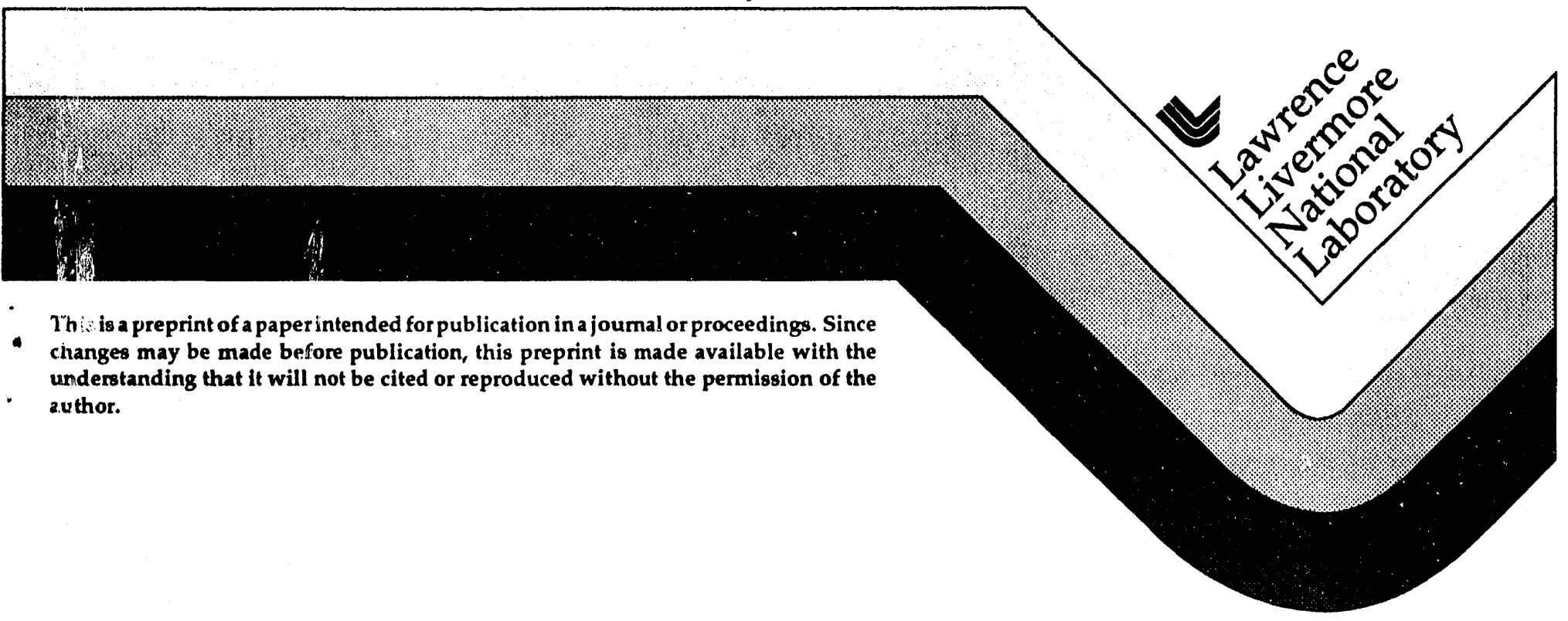

M. M. 
DISCLAIMER

This document was prepared as an account of work sponsored by an agency of the United States Government. Neither the United States Government nor the Uni versity of California nor any of their employees, makes any warranty, express or implied, or assumes any legal liability or responsibility for the accuracy, completeness, or usefulness of any information, apparatus, product, or process disdosed, or represents that its use would not infringe privately owned rights. Reference herein to any specific commercial products, process, or service by trade name, trademark, manufacturer, or otherwise, does not necessarily constitute or imply its endorsement, recommendation, or favoring by the United States Government or the University of California. The views and opinions of authors expressed herein do not necessarily state or reflect those of the United States Government or the University of Califormia, and shall not be used for advertising or product endorsement purposes. 


\title{
OPACITY MEASUREMENTS IN SHOCK-GENERATED ARGON PLASMAS
}

\author{
David Erskine
}

\author{
Lawrence Livermore National Laboratory, PO Box 808 L-299, Livermore, CA 94550
}

\begin{abstract}
Dense plasmas having uniform and constant density and temperature are generated by passage of a planar shock wave through gas. The opacity of the plasma is accurately measured versus wavelength by recording the risetime of emitted light. This technique is applicable to a wide variety of species and plasma conditions. Initial experiments in argon have produced plasmas with $2 \mathrm{eV}$ temperatures, $0.004-0.04 \mathrm{~g} / \mathrm{cm}^{3}$ densities, and coupling parameters $\Gamma \sim 0.3-0.7$. Measurements in visible light are compared with calculations using the HOPE code. An interesting peak in the opacity at $400 \mathrm{~nm}$ is observed for the first time and is identified with the $4 \mathrm{~s}-5 \mathrm{p}$ transition in excited neutral argon atoms.
\end{abstract}

\section{INTRODUCTION}

Opacities of dense plasmas, both theoretically calculated and experimentally measured are an important area of study 1 . For example in astrophysics, the radial structure of the sun is determined by the balance between gravitational forces and a wavelength, density and temperature dependent opacity of the stellar plasma. In laser fusion, the opacity determines the coupling of laser light to drive the target compression. Opacity models are complex requiring much detailed knowledge of the atomic structure, level populations, spectral line shapes and of plasma interactions. Thus the opacity can be a stringent test of our understanding of plasma behavior.

Figure 1 taken from ref. 2 maps the validity domains of the equation of state models of $\mathrm{Al}$. Note that the shaded region at the center of the figure is not covered by any model. This difficult regime has near solid-like densities and partially ionizing temperatures. The situation for other elements is qualitatively similar. For densities either much greater or less than solid-like the Thomas-Fermi approximation or the isolated atom picture can be used respectively. For low temperatures solid state theories apply. For high temperatures matter is highly ionized, the thermal energy dominates over Coulombic energy, and an ionization equilibrium model is appropriate. However, for partially ionized plasmas near solid-like density we have the worst of all worlds and no model does well.

Because of the complexity of plasma behavior in general, simplifying assumptions are used to make calculations tractable. Thus for all regimes it is important to validate models with experimental data. This need is especially acute for the particular regime of solid-density partially ionized plasmas where no model may exist. Fortunately, this complex regime of

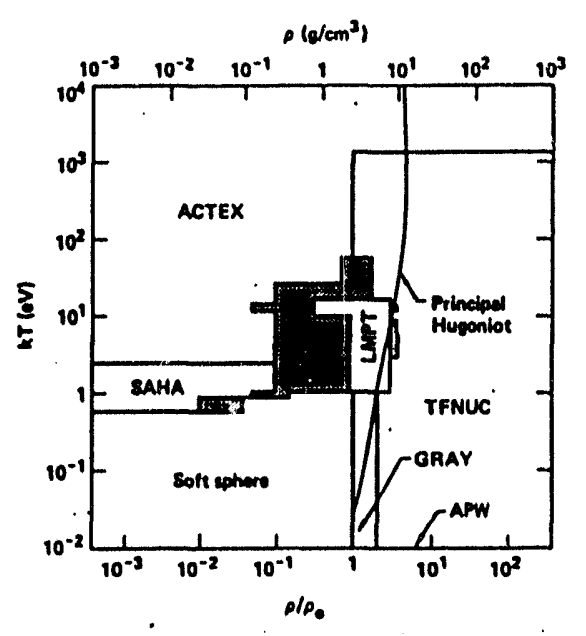

Figure 1. Models used in the Al equation of state, from ref. 2. In the central shaded regions, none of the models is valid. TFNUC: Thomas-Fermi model, ACTEX: ionization equilibrium plasma model, LMPT: liquid metal perturbation theory.

matter is accessible experimentally by shock compression of gases or pulsed laser heating of condensed matter. Because the opacity depencis on many details of plasma behavior it can be used as a stringent test of our understanding.

A primary concern of an opacity experiment is to characterize well the state of the plasma being measured. In addition to the opacity, the density, pressure and temperature need to be accurately determined so that a complete comparison can be made to theory. This can be difficult, for example, in experiments where the plasma is created by a short (ns) laser pulse ${ }^{3}$, because the plasma density decreases as the plasma expands. In addition, the deposited energy distribution may be volumetrically non-uniform. However, the shock wave from the 
impact of a planar projectile into gas provides an ideal method for generating - well-characterized plasma and simultaneously accurately measuring the equation of state (EOS) and opacity. The shock wave generated plasma is intrinsically uniform over a large area (several $\mathrm{cm}$ in diameter) and has a state $(\rho, P, T)$ that for a simple shock is steady in time as it grows in volume. The thickness of the shock front is usually negligible and the relaxation mechanisms through the shocked volume are much faster than the observation rate, so that local thermal equilibrium can be assumed.

In this method the thickness of the plasma is well defined and grows linearly with time, allowing the opacity to be obtained easily from the risetime of the emitted light. Using emitted light rather than transmitted light to determine opacity is an advantage, since in the transmission method an illumination source brighter than the plasma must be found and a separate reference beam from that source measured during the experiment.

A good review of dynamic methods in plasma physics is by Fortov4. Early shock plasma experiments to measure the equation of state of gases 5,6 were performed using an optical technique similar to what we describe below. However the emission risetime was not interpreted into an opacity. The first explicit opacity measurements were performed by Fortov et al. ${ }^{7}$ in the late 1970's on argon, hydrogen-argon and xenon plasmas using explosively driven projectiles and measured emission simultaneously at 1-3 wavelengths. We have improved the technique by increasing the temporal resolution and number of wavelength channels

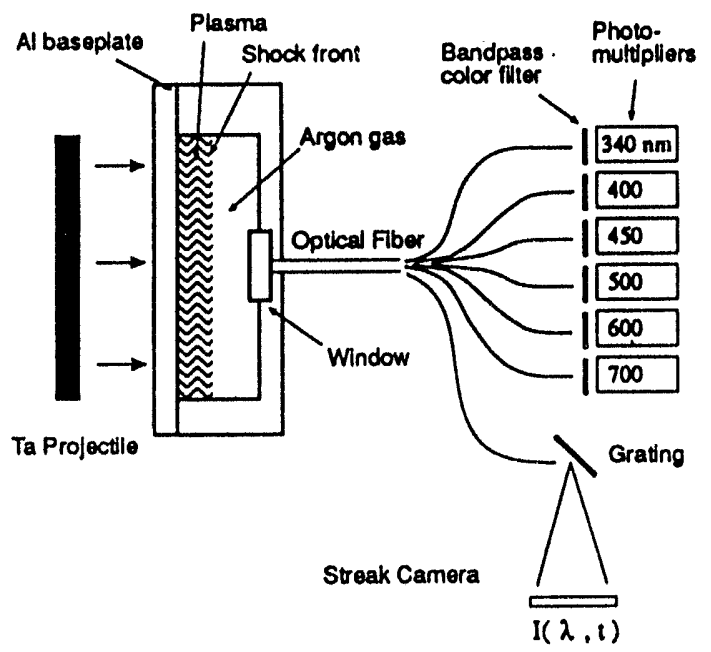

Figure 2. Experimental Arrangement. The Ta impactor disk generates a shock which propagates through Al baseplate into argon gas. Light emitted from the plasma travels through optical fibers to be recorded by photomultipliers with color filters, or a streak camera that measures the time dependent spectrum.

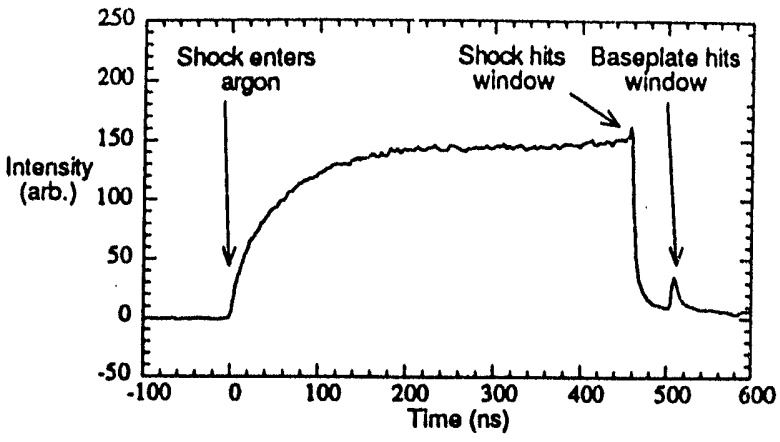

Figure 3. Incandescence at $400 \mathrm{~nm}$ from shocked argon plasma showing exponential-like rise to an asymptotic level, and the arrivals of the shock wave and baseplate at the window at the rear of the chamber. At the arrivals, the increase of light due to re-shock is contemporaneous with increasing attenuation from window damage.

through the use of a streak camera/diffraction grating, and by using gas-gun-accelerated projectiles to produce well-controlled states. Extension of the detection from the visible into the IR and vacuum ultraviolet is planned. The technique is applicable to a wide variety of gas species, and can create plasmas having a large range of densities and temperatures.

Our initial experiments are on argon because its equation of state and opacity model are theoretically well understood. Using a single $\mathrm{Al}$ baseplate and $\mathrm{Ta}$ projectile at $5 \mathrm{~km} / \mathrm{s}$ we can create in 1 bar argon gas a $2 \mathrm{eV}$ plasma with $\rho=0.016 \mathrm{~g} / \mathrm{cm}^{3}$, $\mathrm{P} \sim 1 \mathrm{kbar}$ and $\Gamma=0.5$. $\Gamma=(\mathrm{Ze})^{2} /<\mathrm{a}>\mathrm{kT}$ is the ratio between the coulombic and thermal energies where $<a>$ is the average inter-ion separation. $\Gamma$ near unity describes a strongly coupled plasma. Greater initial gas densities will produce greater plasma densities. Using a multi-layered baseplate for improved kinetic coupling and a Ta projectile velocity of $7.8 \mathrm{~km} / \mathrm{s}$ we expect to produce in 50 bars of argon a plasma that has near solid-like density, but at extreme temperature: $\rho=0.7 \mathrm{~g} / \mathrm{cm}^{3}$ and $\mathrm{T}=6 \mathrm{eV}$. The shock speed, mass velocity and plasma temperature all can be determined optically. Thus the technique of generating plasmas through shock waves accesses an extreme regime of matter under well characterized conditions over a macroscopic volume.

\section{EXPERIMENTAL METHOD}

Figure 2 shows the experimental arrangement. A cylindrical chamber encloses gas having an initial pressure 0 - 50 bar. A two stage gas gun accelerates a tantalum disk projectile to speeds up to $7.8 \mathrm{~km} / \mathrm{s}$. Impact of the disk on the baseplate generates a planar shockwave that enters the chamber, heating the gas to incandescence. Light from the plasma is conducted 
through optical fibers to the detectors. These consist of either a set of photomultipliers, each with a bandpass filter, or a streak camera recording the time dependent spectrum dispersed by a grating. A CCD camera digitally stores the streak camera record and digitizers record the photomultiplier outputs.

Figure 3 is an example of the emission at $400 \mathrm{~nm}$. The entrance of the shock in the chamber is marked by the appearance of light. The arrivals of the shock and the baseplate at the window at the chamber rear is marked by the signal discontinuities at $\sim 460$ and $\sim 510 \mathrm{~ns}$. These transit times provide a measure of the shock $\left(U_{\mathrm{S}}\right)$ and plasma mass velocities $\left(\mathrm{U}_{\mathrm{p}}\right)$.

The opacity is determined from the shape of the emission rise. The plasma volume has the shape of a slab whose thickness increases linearly with time at the shock speed minus the baseplate velocity. For a given wavelength $\lambda$ the incandescence (I) grows from zero to an asymptotic value $\left(\mathrm{I}_{0}\right)$ with a risetime determined by the plasma opacity $(\sigma)$. In the case where baseplate reflectivity is neglected, the time dependent emission follows

$$
\frac{I}{I_{0}}(\lambda)=1-e^{-\sigma(\lambda) \rho\left[U_{s}-U_{P}\right] \Delta l}
$$

where $\rho$ is the plasma mass density and $\Delta t$ is the time after the shock enters the argon. Since the shock density is related to the shock and baseplate speeds by $\rho / \rho 0=U_{S} /\left(U_{S}-U_{p}\right)$, the baseplate velocity can be factored from $\mathrm{Eq} .1$ :

$$
\frac{I}{I_{0}}(\lambda)=1-e^{-\sigma(\lambda) \rho_{0} U_{s} \Delta t}
$$

Thus the baseplate velocity and plasma density need not be known to compute $\sigma$. The effect of parallel reflective surfaces of the chamber is to increase the apparent plasma thickness for early times when the plasma slab is optically thin. If $R_{1}$ and $R_{2}$ are the reflectivities of the baseplate and rear wall respectively then the general expression is

$$
\frac{I}{I_{0}}(\lambda)=[1-A] \frac{\left[1+R_{1} A\right]}{\left[1-R_{1} R_{2} A^{2}\right]}
$$

where

$$
A=e^{-\sigma(\lambda) \rho_{0} U_{S} \Delta t}
$$

For our target $R_{2}=0.7$. In an independent experiment we have measured $R_{1}$ to be $\sim 0.4$ under these shock conditions.

The opacity is found by fitting the light emission to Eq. 3. The plasma density and pressure are determined from $U_{s}$ and $U_{p}$ using elementary shock relations ${ }^{8}$ conserving momentum and mass across the shock front: $\mathrm{P}=\rho_{0} \mathrm{U}_{\mathrm{s}} \mathrm{U}_{\mathrm{p}}$ and $\rho / \rho_{0}=\mathrm{U}_{\mathrm{s}} /\left(\mathrm{U}_{\mathrm{s}^{-}}\right.$ $\left.U_{p}\right)$. The temperature is measured by fitting $I_{0}(\lambda)$ to a greybody emitter. Thus the plasma opacity and its complete EOS can be determined solely from the light emission and the known initial gas density.

\section{RESULTS}

The measured opacities of two shots having 0.3 bar and 3 bar initial argon pressures are compared to calculated results using the $\mathrm{HOPE}^{9}$ code in Fig. 4. The HOPE code calculates opacities by solving the selfconsistent average atom problem and by using firstorder perturbation theory to calculate levels and lines in the detailed configuration model. The overall decrease of $\sigma$ with photon energy from 1 to $7 \mathrm{eV}$ is due to free-free transitions (bremsstrahlung). The

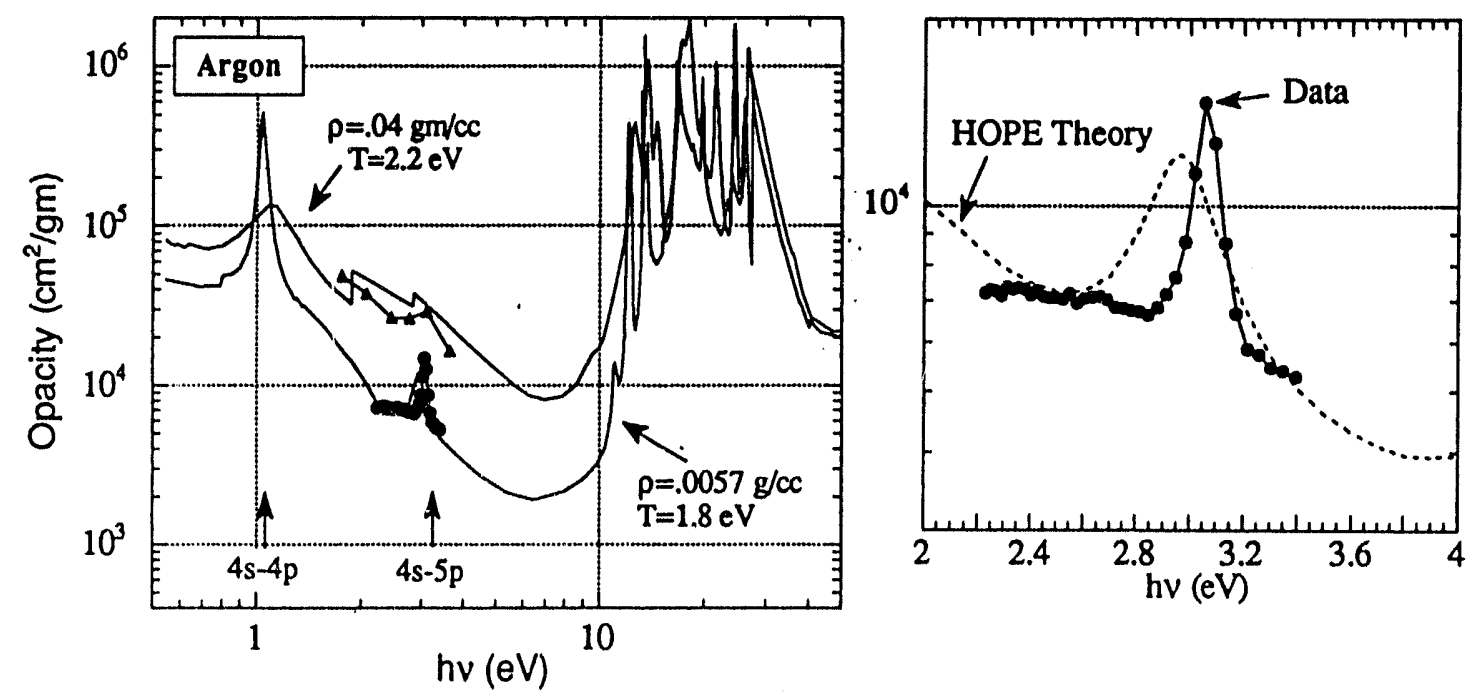

Figure 4. Measured and calculated opacities versus photon energy for two plasma densities: triangles $-0.0057 \mathrm{~g} / \mathrm{cm}^{3}$; circles $-0.04 \mathrm{~g} / \mathrm{cm}^{3}$. Figure on the right is a closeup of the opacity peak in the higher density plasma. Theoretical curves are calculated with the HOPE ${ }^{9}$ code. 


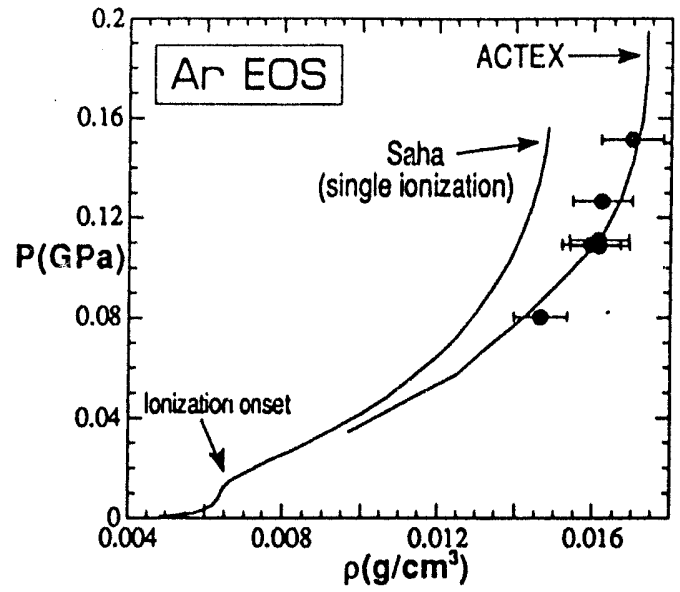

Figure 5. Hugoniot data for shock-generated argon plasma compared with a simple single ionization model (Saha) and a more comprehensive model (ACTEX ${ }^{10}$ ).

peak at $\sim 3 \mathrm{eV}$ is due to a $4 \mathrm{~s}-5 \mathrm{p}$ transition of neutral argon atoms. The forest of peaks above $10 \mathrm{eV}$ are due to deeper-lying bound-bound transitions.

The data of the lower-density plasma (triangles) was recently taken with the streak camera and for the first time clearly shows the lineshape of the opacity peak due to the $4 \mathrm{~s}-5 \mathrm{p}$ transition. The disagreement in linewidth between the theory and experiment is under investigation. It is likely related to approximations in the code for evaluating collisional broadening. The higher plasma density data was taken with six photomultiplier wavelength channels and does not resolve the width of the peak.

Figure 5 shows our Hugoniot data for argon at 1 bar initial pressure. The largest uncertainty is in density $\rho / \rho_{0}=U_{s} /\left(U_{s}-U_{p}\right)$, since it depends reciprocally on the difference between two similar numbers. Improving the time resolution of the baseplate arrival time will reduce this uncertainty. Our uncertainty in $U_{s}$ and $U_{p}$ is $<0.5 \%$ so that $P$ is known to better than $1 \%$.

The data is compared with two theories: a simple single ionization Saha model, and the ACTEX 10 code. This data, as well as that of Christian and Yarger ${ }^{5}$ fits ACTEX well. ACTEX is an ionizationequilibrium plasma model which uses effective electron-ion potentials fitted to experimental spectroscopic data. The possibility of strong coupling of ions is included.

\section{SUMMARY}

Measuring the opacity and EOS of plasma generated through shock impact offers significant advantages over other techniques. These include simultaneous measurement of opacity, Hugoniot and temperature, and a plasma state which is steady in time and uniform over a macroscopic volume. The opacity is determined by the plasma emission risetime rather than transmission. Thus a diagnosed and appropriately brighter illumination source is not required. This technique can be applied to a variety of gas species and plasma densities and temperatures. In argon plasma we have discovered and measured for the first time the lineshape of an opacity peak at $400 \mathrm{~nm}$ due to the $4 \mathrm{~s}-5 \mathrm{p}$ transition in the neutral atom.

\section{ACKNOW/LEDGEMENTS}

Thanks to B. Rosznyai and F. Rogers for calculated opacities. Work performed under the auspices of the U.S. Department of Energy by the Lawrence Aivermore National Laboratory under contract number W-7405-ENG-48.

\section{REFERENCES}

[1] Good reviews are found in S. Ichimaru, $H$. Iyetomi, and S. Tanaka, Phys. Rep. 149, 91 (1987); Strongly Coupled Plasma Physics, ed. F.J. Rogers and H.E. DeWitt (Plenum, New York, 1978); Physics of Nonideal Plasma, ed. V.E. Fortov, I.T. Iakubov (Hemisphere Publ. Co., NY, 1990).

[2] D.A. Young, J.K. Wolford, F.J. Rogers, Phys. Lett. 108A, 157 (1985).

[3] A.Mostovych et al., Phys. Reo. Lett. 66,612 (1991); T.S. Perry et al., Phys. Rev. Lett. 673784 (1991); P.T. Springer et al. 693735 (1992).

[4] V. E. Fortov, Sov. Phys. Usp. 25(11), 781 (1982).

[5] R. H. Christian and F.L. Yarger, J. Chem. Phys. 23 (11) 2042 (1955);

[6] V.K. Gryaznov, M.V. Zhernokletov, V.N. Zubarev, I.L. Iosilevskii, and V.E. Tortovk, Sov. Phys. JETP 51 (2), 288 (1980).

[7] V.E. Bespalov, V.K. Gryaznov, and V.E. Fortov, Soo. Phys. JETP 49 71 (1979);

V.E. Fortov, V.E. Bespalov, M.I. Kulish, S.I. Kuz, Strongly Coupled Plasma Physics, S. Ichimaru Ed., Elsevier Science Publ., p571 (1990).

[8] A.C. Mitchell and W.J. Nellis, J. Appl. Phys. 52, 3363 (1981).

[9] B. Rosznyai, Defns. Res. Rev. 1, No. 3, April 1989, p21.

[10] F.J. Rogers, Phys. Rev. A24, 1531 (1981). 

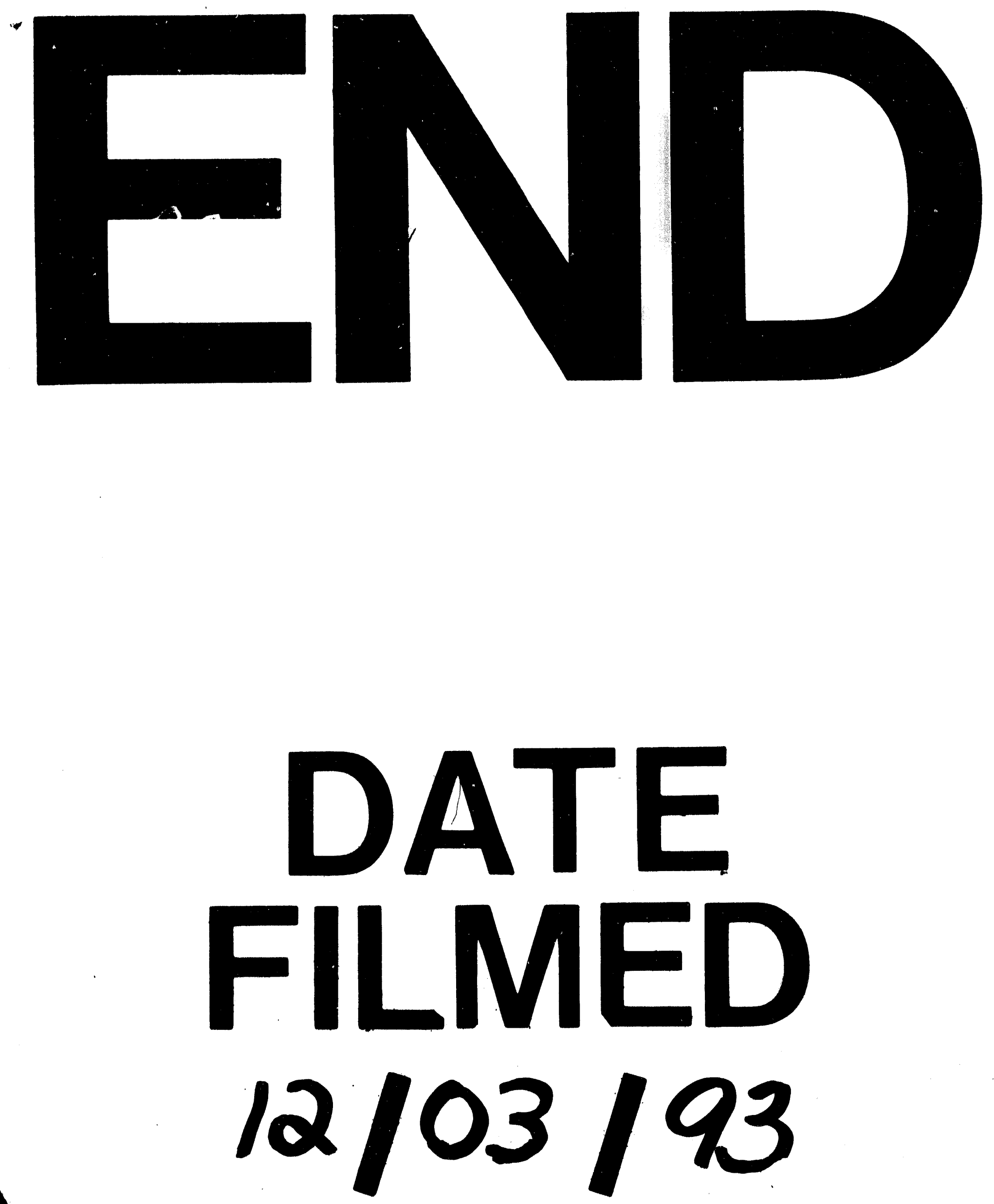
